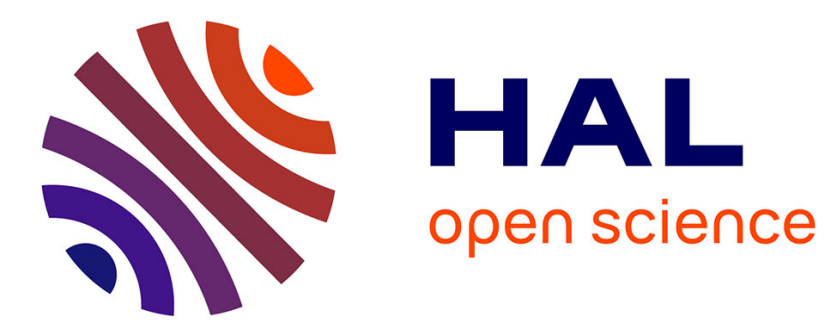

\title{
A study of the temperature effect on photoluminescence of the P3HT/MWNT nanocomposites
}

\author{
Anass Bakour, Omar Bajjou, Mimouna Baitoul, Florian Massuyeau, Jany \\ Wéry, Malik Maaza, Eric Faulques
}

\section{- To cite this version:}

Anass Bakour, Omar Bajjou, Mimouna Baitoul, Florian Massuyeau, Jany Wéry, et al.. A study of the temperature effect on photoluminescence of the P3HT/MWNT nanocomposites. Materials Today: Proceedings, 2021, 36, Part 2, pp.549-552. 10.1016/j.matpr.2020.05.326 . hal-03266188

\section{HAL Id: hal-03266188 \\ https://hal.science/hal-03266188}

Submitted on 16 Jul 2021

HAL is a multi-disciplinary open access archive for the deposit and dissemination of scientific research documents, whether they are published or not. The documents may come from teaching and research institutions in France or abroad, or from public or private research centers.
L'archive ouverte pluridisciplinaire HAL, est destinée au dépôt et à la diffusion de documents scientifiques de niveau recherche, publiés ou non, émanant des établissements d'enseignement et de recherche français ou étrangers, des laboratoires publics ou privés. 


\title{
A study of the temperature effect on photoluminescence of the P3HT/MWNT nanocomposites
}

\author{
Anass Bakour ${ }^{a^{*}}$, Omar Bajjou ${ }^{b}$, Mimouna Baitoul ${ }^{c}$, Florian Massuyeau ${ }^{d}$, Jany Wéry ${ }^{d}$, Malik Maaza ${ }^{e}$, \\ Eric Faulques ${ }^{d}$ \\ a Laboratory of Physics of Condensed Matter and Renewable Energy, Faculty of Sciences and \\ Technology, University of Hassan II Casablanca, PoBox 146, 28806 Mohammedia, Morocco \\ ${ }^{b}$ Material Physics Laboratory, Faculty of Sciences and Technology, University Sultan Moulay Slimane, \\ BP 523, 23000 Beni Mellal, Morocco \\ ${ }^{c}$ Laboratory of Solid state physics, research group polymers and nanomaterials \\ USMBA, Faculty of Sciences Dhar el mahraz, PoBox 1796 Atlas, 30000 Fes, Morocco \\ ${ }^{d}$ Institut des Matériaux Jean Rouxel, Nantes, 2 rue de la Houssinière, BP32229, 44322 Nantes, \\ Cedex3, France \\ ${ }^{e}$ UNISA Africa Chair in Nanosciences-Nanotechnology, College of Graduate Studies, University of \\ South Africa, Muckleneuk Ridge, PO Box 392, Pretoria, South Africa
}

*Corresponding author Email address: bakour.anass@gmail.com

\begin{abstract}
:
In spite of the strong competition from recently developed nanomaterials such as graphene, carbon nanotubes continue to be promising materials for photovoltaic applications thanks to their structural advantages and cheap prices of synthesis especially the multi-walled carbon nanotubes (MWNT). Mixing of MWNT with a semiconducting polymer such as the P3HT leads to a composite material characterized with enhanced mechanical, thermal and optical properties. In order to investigate the impact of the insertion of different wt $\%$ of MWNTs on the luminescence properties of the P3HT we conducted a series of measurements by means of steady state PL and time transient PL for different samples, as well as the study of the temperature effect on these properties. The results obtained confirm the luminescence quenching effect played by the MWNTs, and prove that the lifetime of photo-generated excitons in the P3HT matrix undergoes a modification after the insertion of the MWNTs which means that a charge separation process takes place at the P3HT/MWNT interface.
\end{abstract}

\section{Introduction}

This work is devoted to the exploration of light energy conversion at the interface between carbon nanotubes (CNTs) and semi- conducting polymer poly(3-hexylthiophene) which have proven to have an interesting optical characteristic. In this context, photo-conversion processes comprise light absorption, photo- induced charge transfer, exciton dissociation and photo-carriers transport as a result of absorbed light energy, which are basic photo-physical principles providing functionality of optoelectronic devices such as photo-detectors, OLED and solar cells.

It has been found that the insertion of nanomaterials such as $\mathrm{C}_{60}, \mathrm{CdSe}$, graphene and their derivatives [1-3] with a higher electron affinity into conjugated polymers [4-6] facilitates the charge separation of photo-generated electron-hole pairs at an interface between the polymer and the electron-accepting material. In the bulk hetero-junction efficient charge separation in 
the semiconducting polymer/carbon nanotubes composites can be achieved due to the existence of many interfaces for charge transfer, but the performance of photovoltaic applications is limited by the poor carrier transport in composites after charge separation. Thus, the carbon nanotubes provide an enhanced charge transport path that is required to achieve efficient electronic conduction, thanks to their one dimensional conducting properties and hence offering direct pathways for electric conduction [7]. In this study, we investigated carrier dynamics with respect to charge separation and transport in conjugated P3HT/Multiwalled carbon nanotubes composites by temperature dependent steady state photoluminescence (PL) and time-resolved photoluminescence (TRPL) spectroscopy.

\section{Material and methods}

The regioregular P3HT (RR-P3HT) used in this study (provided from Rieke) has the following characteristics: Average molecular weight $\mathrm{MW}=37.680 \mathrm{~g}$ mol-1, $\mathrm{PDI}=1.48$ (polydispersity) and regio-regularity HT (head-tail)> 98.5\%. Multiwalled carbon nanotubes used in this work were obtained Arkema synthesized by CVD and then purified by $\mathrm{HCl}$.

In our work we used the mixing process in solution, the polymer matrix is first placed in solution in chloroform. The nanotubes on the other hand are dispersed in the same solvent under ultrasonic agitation. The CNT solution is then added to the polymer matrix solubilized under stirring. The nanotubes in this way can be introduced between the macromolecular chains of the polymer. The composite solution is then drop casted on silica substrate and left to dry at $50^{\circ} \mathrm{C}$.

The Steady-state in photoluminescence measurements were performed at different temperatures using a Jobin-Yvon Fluorolog 3 spectrometer using a Xenon lamp (500 W) and a CCD detector, temperature was controlled by a cryostat coupled with the Fluorolog cooled by liquid nitrogen.

Time-resolved photoluminescence (TR-PL) experiments were performed with a regenerative amplified femtosecond Ti:Sapphire laser system (Spectra Physics Hurricane X). This setup generates $100 \mathrm{fs}$ pulses at $800 \mathrm{~nm}$ with a repetitive rate of $1 \mathrm{kHz}$ and a nominal power of $1 \mathrm{~W}$. The laser line is frequency-doubled with a thin BBO crystal to obtain an excitation $\lambda_{\text {exc }}=400$ $\mathrm{nm}$. The pump energy pulse is controlled by means of neutral density filters $(0.1 \mathrm{~mJ}$ with diameter laser beam of $5 \mathrm{~mm}$ falling on the sample) to ensure that the excitation density on the sample do not exceed $1017 \mathrm{~cm}^{-3}$ and to avoid bimolecular annihilation process and sample photo-degradation. Emission spectra are temporally resolved with a high dynamic range Hamamatsu C7700 streak camera coupled to an imaging spectrograph with a temporal resolution less than $20 \mathrm{ps}$ and processed using the HPDTA Hamamatsu software.

\section{Results and discussions}

\subsection{Steady state photoluminescence}


The effect of temperature on the steady state PL is studied because of the strong relation between the electronic band gap of P3HT/MWNT composites and determining the role of the electron-phonon interactions [8].

To investigate the transport and luminescence mechanism of the P3HT/MWNT composite, steady state photoluminescence was measured as a function of temperature within the range of $\left[90^{\circ} \mathrm{K}, 300^{\circ} \mathrm{K}\right]$, by a difference of $40^{\circ} \mathrm{K}$ between each measure controlled by means of a cryostat with liquid nitrogen, at an excitation wavelength of $420 \mathrm{~nm}$.

P3HT light absorption is an initial step leading to the generation of bound excitons. In order to convert light energy into an electrical signal, the excitons should be separated into free charge carriers (electrons and holes), at the P3HT/MWNT interface. Theoretically, the absorption and emission spectra are symmetrical with respect to the energy of the zero-phonon band for which light emission takes place at the highest energy [9]. The P3HT photoluminescence spectrum at room temperature showed in Fig. 1(a) exhibits usually two peaks. In the literature, these two peaks are commonly assigned to the transitions between the vibrational energy levels (0-0) and (0-1). The (0-0) peak at $664 \mathrm{~nm}(1.86 \mathrm{eV})$ is often less intense than the $(0-1)$ peak centered at $715 \mathrm{~nm}(1.73 \mathrm{eV})$. How- ever, the zero-phonon line is usually in real systems the most intense line and it is consistent with results obtained by other researchers [10].
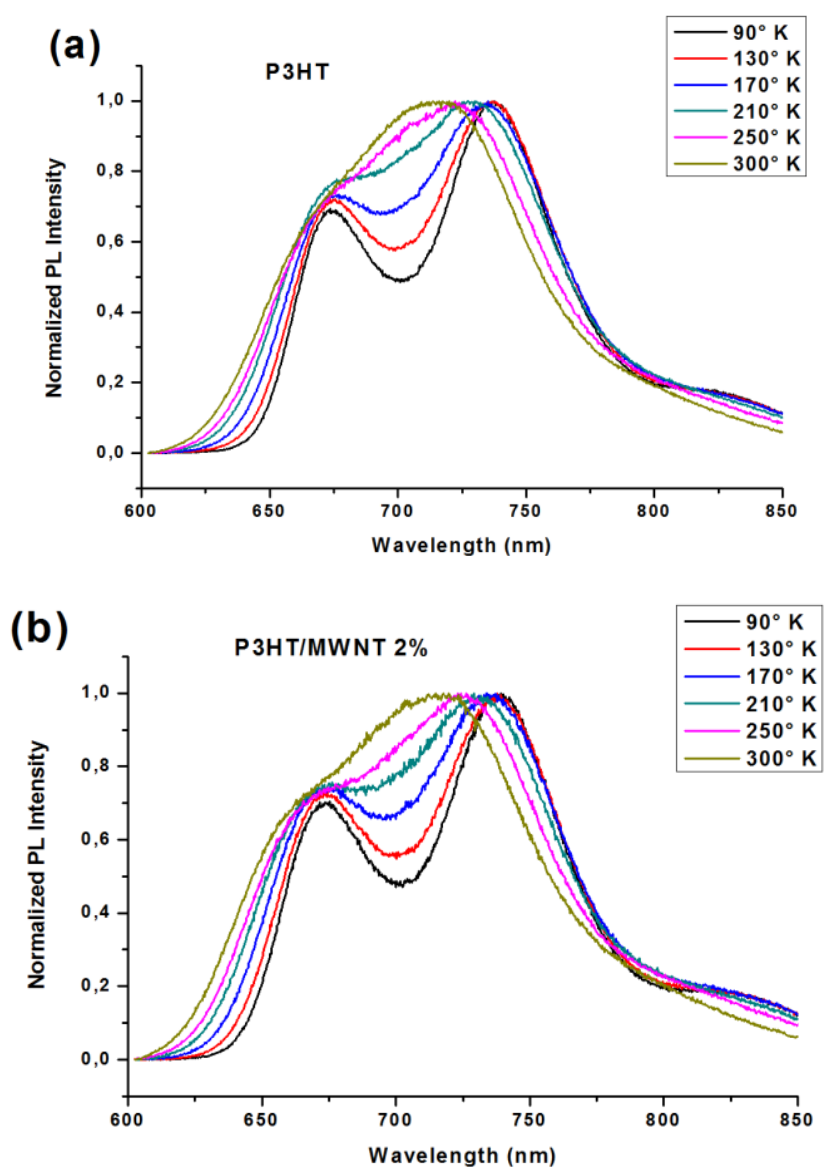

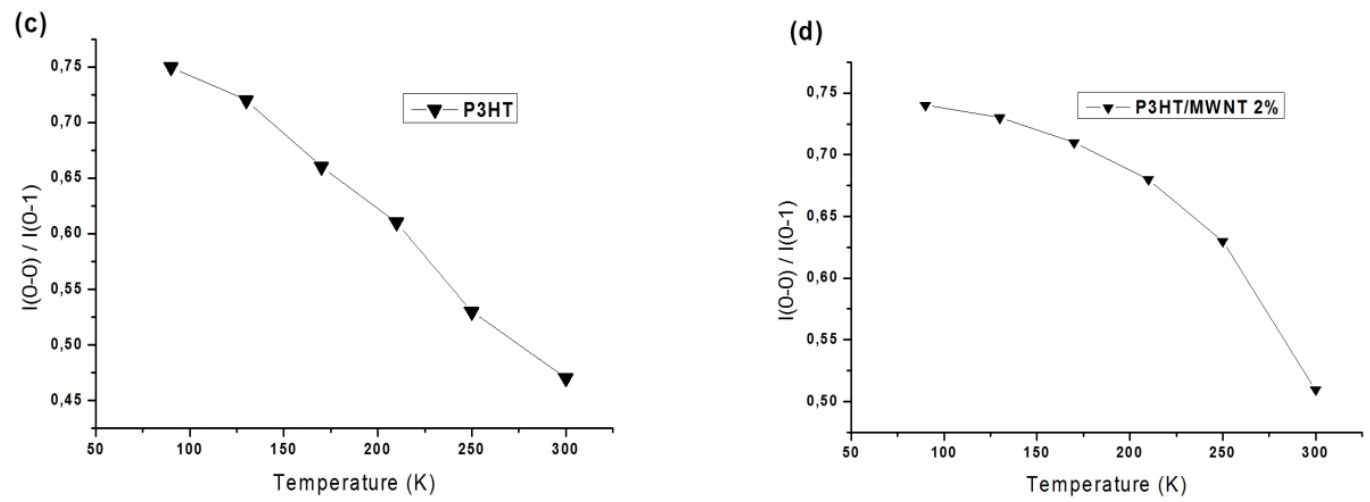

Fig. 1: Temperature dependent Normalized PL spectra of : (a) P3HT film. (b) P3HT/MWNT $2 \mathrm{wt} \%$ film. Intensity ratio of the (0-0) to the (0-1) peaks as a function of temperature of (c) P3HT, (d) P3HT/MWNT 2wt\%

In Fig. 1(a) we notice an overall gradual redshift in the spectrum of P3HT, especially in the (0-1) peak that shows a strong shift to $740 \mathrm{~nm}$ when the temperature decreases to $90^{\circ} \mathrm{K}$, and a less pronounce redshift in the (0-0) peak to $674 \mathrm{~nm}$. Along with this shift, we note a change in the relative intensity of the two characteristic peaks while varying the temperature gradually. In order to give a better insight of this effect we exhibit in the Fig. 1(c) the intensity ratio of the $0-0$ band to the (0-1) band as a function of the temperature, this ratio is related to the effective Huang-Rhys parameter of the emitting state [11,12], in this plot we can see that the value of $\mathrm{I}_{(0-0)} / \mathrm{I}_{(0-1)}$ decreases from 0.75 at a $90^{\circ} \mathrm{K}$ to 0.47 at room temperature.

In Fig. 1(b) we show the progress of the P3HT/MWNT composite film PL spectrum at $2 \mathrm{wt} \%$ of MWNT, as a function of temperature in the same range used for the pristine P3HT film. The overall trend of the spectrum is to move toward lower energy confirming the redshift already noted for the P3HT sample. The (0-0) peak at room temperature shows a slight shift to $657 \mathrm{~nm}$ when compared to the P3HT spectrum, this can be attributed to an increased percentage of short segments over long segments in the matrix owing to the wrapping effect of the P3HT over the MWNTs [13,14]. Although, this shift doesn't persist when cooling the samples, we notice that the two bands start to have the same respective positions for both samples due to the reduction in thermal disorder in predominant component which is the polymer. The main peaks intensity ratio for the P3HT/MWNT $2 \mathrm{wt} \%$ is shown in Fig. 1(d) exhibiting a decrease from 0.74 at $90^{\circ} \mathrm{K}$ to 0.51 at room temperature confirming the tendency of a more ordered regions and enhanced conjugation length of the polymer chains by reducing the conformational disorder. The excited states are therefore delocalized over the more conjugated polymer chain, giving lower-energy emission.

It is observed that the normalized PL spectra of P3HT and composite films P3HT/MWNT at 2 wt\% of MWNT show a slight shift when compared on the concentration base at room temperature, except the effect of quenching confirming the role of the MWNT in favoring the exciton dissociation at the P3HT/MWNT interface. The extinction of the photoluminescence could also be due to the presence of aggregates in the composite material, or due to the existence of some defects on the surface of carbon nanotubes. This effect is also observed 
when cooling the samples and thus confirming the elimination of the phonons contributing to the non radiative recombination in the samples.

\subsection{Time Transient Photoluminescence}

The luminescence decay of the excited states involves two com- peting processes, radiative and non-radiative processes. The rela- tive proportion of these processes determines the efficiency of luminescence. If the non-radiative channel is very fast, a significant portion of photogenerated excitations will decline through that pathway before having the time of issuing radiatively.

The results found in the Steady state PL hereinbefore suggesting enhanced electronic delocalization in the composite films can be further investigated by the time-resolved PL analysis.

Fig. 2 Shows the time resolved PL of the P3HT and P3HT/MWNT 2 wt\% composite films at room temperature, at an excitation wave- length of $420 \mathrm{~nm}$. The fitting of the decay patterns of the P3HT and P3HT/MWNT 2 wt\% composite is shown in Fig. 2(b) and (c) respectively, using a bi-exponential decay model $[15,16]$.
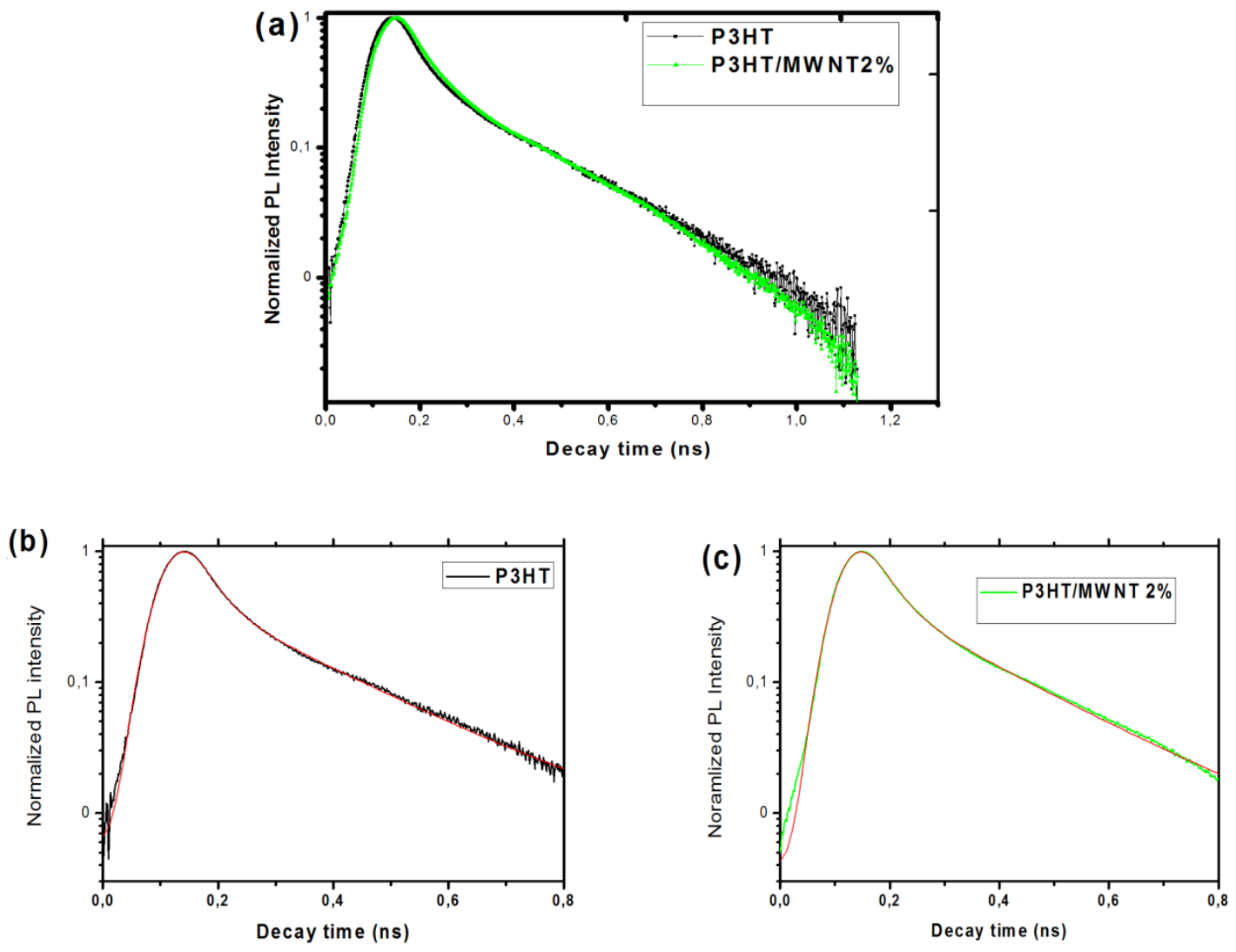

Fig.. 2 TRPL emission decays of: (a) P3HT and P3HT/MWNT 2wt\% composite film. And decay fitting of (b) P3HT, (c) P3HT/MWNT 2wt $\%$

It is observed in Table 1 that the mean lifetime $\tau_{\text {mean }}$ exhibits a decrease when inserting a 2 wt $\%$ concentration of nanotubes in the P3HT, suggesting that the decline is slightly faster for 
the $2 \mathrm{wt} \%$ composite. The decreasing PL lifetime is attributed to efficient charge separation from the increase of non-radiative pathway created by the insertion of MWNT. The remaining non recombined excitons which contribute to the remaining luminescence in polymer will migrate over more conjugated region before recombination [17].

\begin{tabular}{|l|c|c|c|c|c|}
\hline Sample & $\tau_{1}(\mathrm{ps})$ & $\mathrm{A}_{1}$ & $\tau_{2}(\mathrm{ps})$ & $\mathrm{A}_{2}$ & $\tau_{\text {mean }}(\mathrm{ps})$ \\
\hline P3HT & 29 & 2,60 & 196 & 0,56 & 134 \\
\hline $\begin{array}{l}\text { P3HT/MWNT } \\
\text { 2wt\% }\end{array}$ & 34 & 2,39 & 194 & 0,64 & 115 \\
\hline
\end{tabular}

Table. 1: PL decay times $\left(\tau_{1}, \tau_{2}, \tau_{\text {mean }}\right)$, pre-exponential factor $\left(\mathrm{A}_{1}, \mathrm{~A}_{2}\right)$ of the P3HT and P3HT/MWNT 2\%wt composite films

We can therefore conclude that there is indeed a notable dissociation of charges due to the presence of nanotubes that causes the appearance of a greater probability of non-radiative process. Moreover, the contribution of rapid exponential first became prominent in the decline and thus the lifetimes decrease. This separation is made possible by the migration of excitations from short segments to long segments. During this migration, a significant fraction of excitations hops on the MWNT network on which they dissociate non-radiatively [13]. In future, it is intended to optimize the current system in view of validating its broad white luminescence and other potential application [18-20].

\section{Conclusion}

In this work we have conducted an investigation on the photo- luminescence and the charge separation processes in the P3HT and P3HT/MWNT composite as well as the effect of temperature on these properties. It has been demonstrated by means of steady state PL that the insertion of MWNT in the P3HT matrix leads to an efficient PL quenching due to the nucleant agent role played by MWNTs, as well as the phonon contribution to the luminescence that was investigated by varying measurements temperature. This result reveals is the characters of enhanced short segment conjugation over long segments in the polymer/MWNT composite systems. Exciton dissociation and migration due to enhanced electronic delocalization in polymer after adding MWNT was demonstrated by TRPL measurements and thus confirming the charge separation at the P3HT/MWNT interface. These effects are of crucial importance for the application of the studied composites in heterojunction organic photovoltaic cells.

\section{Declaration of Competing Interest}

The authors declare that they have no known competing finan- cial interests or personal relationships that could have appeared to influence the work reported in this paper. 
This research was partially supported by the Institute of Mate- rials Jean Rouxel Nantes, France. We thank our colleagues from IMN who provided Materials, insights and expertise that greatly assisted the research, and for their comments that greatly improved the manuscript.

\section{References}

[1] W.U. Huynh, J.J. Dittmer, A.P. Alivisatos, Hybrid Nanorod-Polymer Solar Cells, Science, 295 (2002) 2425-2427.

[2] S.R. Cowan, A. Roy, A.J. Heeger, Recombination in polymer-fullerene bulk heterojunction solar cells, Physical Review B, 82 (2010) 245207.

[3] R. Saito, M. Fujita, G. Dresselhaus, M.S. Dresselhaus, Electronic structure of chiral graphene tubules, Applied Physics Letters, 60 (1992) 2204-2206.

[4] W. Barford, Electronic and Optical Properties of Conjugated Polymers, Oxford University Press, 2013.

[5] O. Bajjou, A. Bakour, M. Khenfouch, M. Baitoul, E. Faulques, M. Maaza, Charge carrier dynamics and $\mathrm{pH}$ effect on optical properties of anionic and cationic porphyrin-graphene oxide composites, J. Electron. Mater. 47 (2018) 2897-2904.

[6] O. Bajjou, A. Bakour, M. Khenfouch, M. Baitoul, E. Faulques, M. Maaza, Synthesis and opto-structural characterization of reduced graphene oxide and meso-tetrakis(4phenylsulfonic-acid) porphyrin composites, J. Mater. Sci.: Mater. Electron. 29 (2018) 85948600 .

[7] P.G. Collins, M.S. Arnold, P. Avouris, Engineering carbon nanotubes and nanotube circuits using electrical breakdown, Science 292 (2001) 706-709.

[8] R.B. Capaz, C.D. Spataru, P. Tangney, M.L. Cohen, S.G. Louie, Temperature dependence of the band gap of semiconducting carbon nanotubes, Phys. Rev. Lett. 94 (2005) 036801.

[9] T. Guillet, J. Berréhar, R. Grousson, J. Kovensky, C. Lapersonne-Meyer, M. Schott, V.

Voliotis, Emission of a single conjugated polymer chain isolated in its single crystal monomer matrix, Phys. Rev. Lett. 87 (2001) 087401.

[10] J. Clark, J.-F. Chang, F.C. Spano, R.H. Friend, C. Silva, Determining exciton bandwidth and film microstructure in polythiophene films using linear absorption spectroscopy, Appl.

Phys. Lett. 94 (2009) 163306.

[11] W. Barford, O.R. Tozer, Theory of exciton transfer and diffusion in conjugated polymers, J. Chem. Phys. 141 (2014) 164103.

[12] F.C. Spano, H. Yamagata, Vibronic coupling in J-aggregates and beyond: a direct means of determining the exciton coherence length from the photoluminescence spectrum, J. Phys.

Chem. B 115 (2011) 5133-5143.

[13] M. Baghgar, J.A. Labastide, F. Bokel, R.C. Hayward, M.D. Barnes, Effect of polymer chain folding on the transition from $\mathrm{H}$ - to J-aggregate behavior in P3HT nanofibers, J. Phys. Chem. C 118 (2014) 2229-2235. 
[14] M. Khenfouch, O. Bajjou, M. Baitoul, M. Maaza, Optical properties and dynamics excitation relaxation in reduced graphene oxide functionalized with nanostructured porphyrins, Opt. Mater. 42 (2015) 479-483.

[15] F. Massuyeau, E. Faulques, H. Athalin, S. Lefrant, J.L. Duvail, J. Wery, E. Mulazzi, R. Perego, Steady state and transient photoluminescence in poly-p-phenylene vinylene films and nanofibers, J. Chem. Phys. 130 (2009) 124706.

[16] A. Bakour, F. Geschier, M. Baitoul, M. Mbarek, K. El-Hadj, J.-L. Duvail, S. Lefrant, E. Faulques, F. Massuyeau, J. Wery-Venturini, Effects of single-walled carbon nanotubes on the optical and photo-conductive properties of their composite films with regio-regular poly(3hexylthiophene), Mater. Chem. Phys. 143 (2014) 1102-1110.

[17] P.J. Goutam, D.K. Singh, P.K. Iyer, Photoluminescence quenching of poly(3hexylthiophene) by carbon nanotubes, J. Phys. Chem. C 116 (2012) 8196-8201. [18] M. Khenfouch, J. Wéry, M. Baïtoul, M. Maaza, Photoluminescence and dynamics of excitation relaxation in graphene oxide-porphyrin nanorods composite, J. Lumin. 145 (2014) 33-37.

[19] M. Maaza, White photoluminescence from a grown $\mathrm{ZnO}$ nanorods/graphene hybrid nanostructure, Opt. Mater. 34 (8) (2012) 1320-1326.

[20] S.O. Oseni, K. Kaviyarasu, M. Maaza, G. Sharma, G. Pellicane, G.T. Mola, ZnO: CNT assisted charge transport in PTB7: PCBM blend organic solar cell, J. Alloy. Compd. 748 (2018) 216-222. 